\title{
ENGEVISTA
}

Página da revista: http://www.uff.br/engevista/seer/

\section{Análise das propriedades de compósitos poliméricos reforçados com fibra de vidro}

Priscilla Rocha Vieira ${ }^{1}$

Janine Vieira $^{2}$

Eliane Maria Lopes Carvalho ${ }^{3}$

Resumo: A proposta deste artigo é apresentar o estudo das propriedades físicas e mecânicas de compósitos poliméricos reforçados com fibra de vidro, assunto de interesse de aplicação na construção civil. Nos últimos 40 anos este material tem sido objeto de estudo em diversas áreas, desde medicina à engenharia. Isto se dá devido suas propriedades favoráveis como, por exemplo, alta resistência mecânica aliada à alta durabilidade. Nesse contexto, foi desenvolvido um programa experimental para análises das propriedades físicas e mecânicas de dois tipos de materiais compósitos, o primeiro reforçado com fibras de vidro particuladas e o segundo com manta de fibras de vidro. Em ambos compósitos foram utilizados a resina epóxi como matriz.

Palavras-chave: Materiais compósitos; fibras particuladas, manta de fibra de vidro, resina epóxi.

\footnotetext{
${ }^{1}$ UFF - Universidade Federal Fluminense

${ }^{2}$ UFF - Universidade Federal Fluminense

${ }^{3}$ UFF - Universidade Federal Fluminense
} 


\begin{abstract}
The aim of this work is to present a study of the physical and mechanical properties of fiber-reinforced polymer (FRP), application of interest issue in civil construction. Over the past 40 years this material has been studied in several areas, from medicine to engineering. This is due to their good properties, such as high mechanical strength combined with high durability. In this context, an experimental program was developed for analysis of physical and mechanical properties of two types of composite materials, in the first were used particulate glass fiber as a reinforced and in the second glass fiber strand mat. In both composites were used as matrix, the epoxy resin.
\end{abstract}

Keywords: Composite materials; particulate fibers, glass fiber strand mat, mechanical properties. 


\section{Introdução}

O material compósito é definido como a combinação de dois materiais quimicamente distintos com uma interface separando os componentes, Broutman [1]. Esses materiais são usados muitas vezes com o objetivo de obter propriedades específicas, diferentes daquelas encontradas isoladamente em cada constituinte. Para o compósito ter um bom desempenho, além do tipo, quantidade e orientação das fibras é necessária uma boa aderência entre os materiais. São materiais, em geral, resistentes, apresentam baixo peso específico, possuem resistência à corrosão, não conduzem eletricidade e algumas matrizes são resistentes ao fogo. Atualmente, são utilizados principalmente na América do norte e Europa, aplicados desde em reforço estrutural a grandes estruturas como pontes, estruturas Off-Shore, torres de telecomunicação e torres meteorológicas.

Os materiais compósitos surgiram a partir dos avanços nos estudos de novos materiais para a indústria de construção civil. A busca por materiais mais leves, duráveis, com alta resistência impulsionaram este setor. Nas últimas décadas, com o desenvolvimento destas novas tecnologias agregaram-se mais segurança, economia e funcionalidade.

Segundo Bank [2], a última década deu um salto de crescimento do uso de materiais compósitos de polímeros reforçados com fibra (FRP- Fiber Reinforced Polymer), na indústria de construção civil. Esses novos materiais estão sendo usados como matéria prima para a construção de pontes e edifícios, assim como para reforços dos mesmos.

Buscando novos conhecimentos em relação ao comportamento dos materiais compósitos, a investigação realizada nesta pesquisa teve como objetivo a comparação de propriedades físicas e mecânicas para dois tipos de compósitos: (i) compósito de matriz polimérica reforçados com fibras de vidro particuladas e (ii) compósito de matriz polimérica reforçadas com manta de fibra de vidro.

\section{Metodologia e Programa experimental}

Em busca de atingir o objetivo desta pesquisa, o programa experimental se dividiu em três etapas:

- Confecção dos corpos de prova;

- Avaliação da massa específica dos compósitos;

- Determinação da resistência à tração dos compósitos.

\subsection{Confecção dos corpos de prova}

Os corpos de prova foram confeccionados utilizando resina epóxi como matriz. Tanto no reforço particulado ou na forma de manta, foram utilizadas fibras de vidro do tipo E-Glass. 
Os corpos de prova utilizados na avaliação da resistência a tração foram fabricados com base na norma ISO 574 [3]. Vale destacar que a espessura do corpo de prova foi determinada de maneira a se obter pelo menos duas camadas de manta de fibra de vidro. Assim, a dimensão final do corpo de prova era $300 \times 25 \times 10 \mathrm{~mm}$.

A partir dos corpos de prova de tração, foram retiradas amostras para a avaliação da massa específica.

Para a confecção dos corpos de prova se fez necessário à utilização de moldes, que no estudo foram fabricados em silicone, material escolhido pela facilidade de desmoldagem dos corpos de prova.

Primeiramente foram realizados corpos de prova somente com a matriz, isto é, nenhum tipo de reforço foi utilizado, ver Figura 1. Esses corpos de prova serviram de referência, para posterior verificação da eficiência dos dois tipos de reforços.

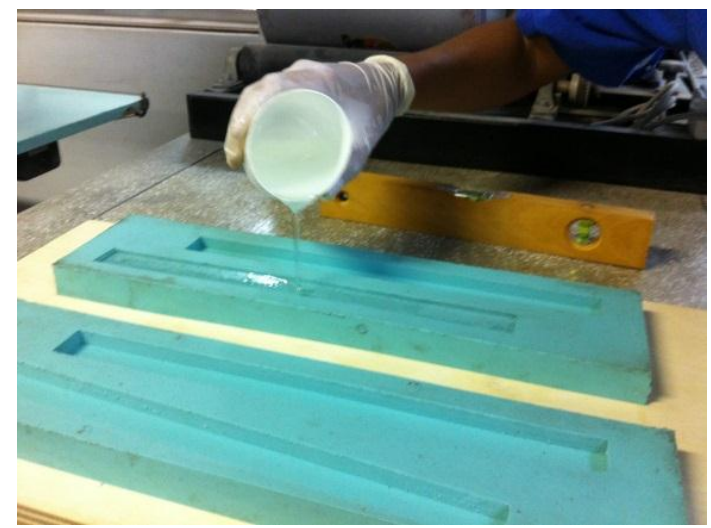

(a) Moldagem dos corpos de prova e referência.

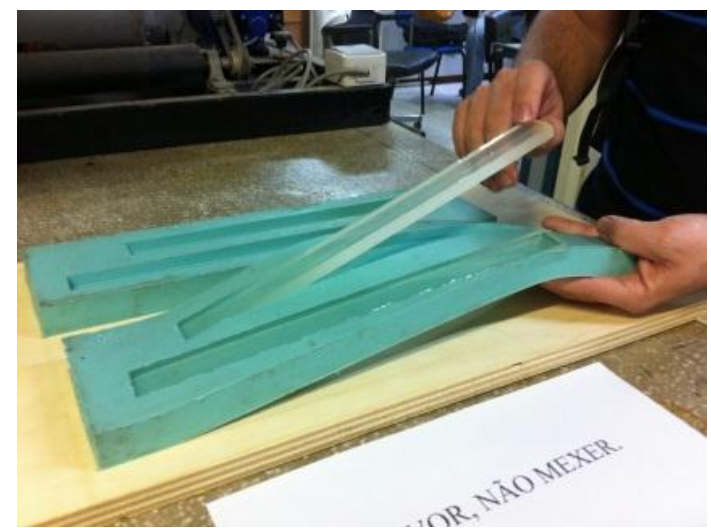

(b) Desmoldagem dos corpos de prova de referência.

Figura 1. Fabricação dos corpos de prova de referência.

\section{Fabricação dos corpos de prova com mantas de fibras de vidro.}

Em função da espessura prevista dos corpos de prova, foi possível a fabricação de corpos de prova com duas e três camadas de manta de fibra de vidro. A manta utilizada na fabricação dos corpos de prova é apresentada na Figura 2a. Os cortes das camadas de manta foram feitos de 
acordo com as dimensões do molde. Após cortadas foram testadas nos moldes para possíveis desvios de medição. Quando necessário, a peça foi recortada novamente até encaixar com precisão. Na Figura $2 \mathrm{~b}$ é apresentado o corte das camadas de manta.

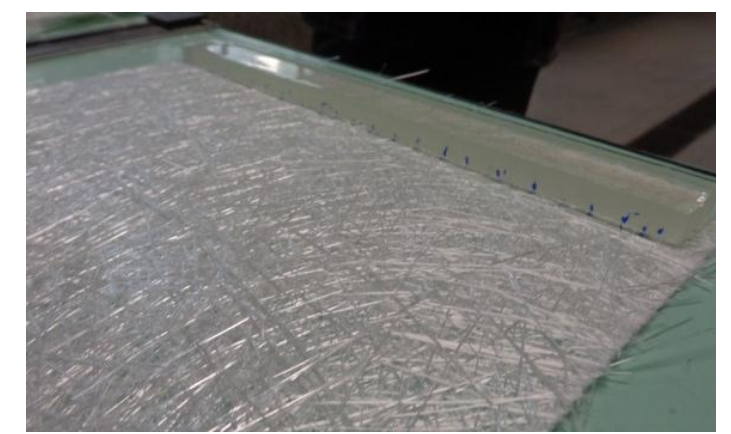

(a) Manta utilizada na fabricação dos compósitos.

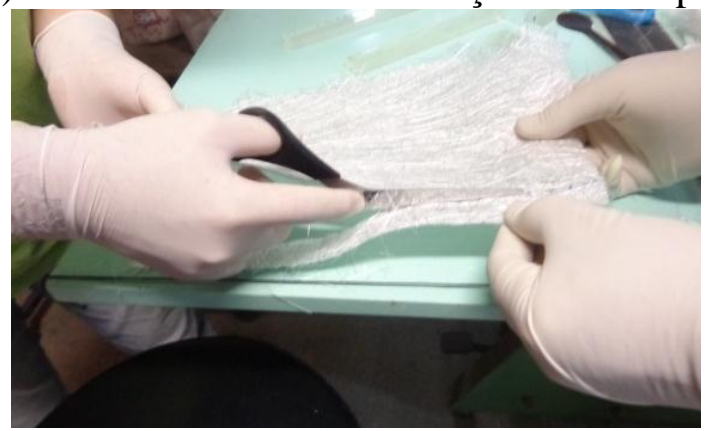

(b) Corte da camada de manta

Figura 2. Preparação das camadas de manta

Após os cortes das camadas cada camada de manta foi pesada a fim de se obter a massa de fibra utilizada em cada corpo de prova. Para a pesagem, foi utilizada uma balança da marca Marte AS2000C, com sensibilidade 0.01g.

Inicialmente foi colocada uma camada de matriz, seguida da primeira camada de manta e cuidadosamente procedeu-se a nova camada de matriz para a total impregnação da manta de fibra de vidro. O procedimento se repetiu até a última camada de manta.

Após 12 horas de cura, ocorreu a desmoldagem dos corpos de prova. Estes foram lixados, para eliminar filamentos de fibras, pesados e medidos (comprimento, largura e espessura).

\section{Fabricação dos corpos de prova com fibras de vidro particuladas.}

Para a fabricação dos corpos de prova com fibras particuladas, as fibras foram cortadas com comprimento de aproximadamente $15 \mathrm{~mm}$. Como parte do procedimento experimental foi definida que a massa de fibra particulada seria igual à massa das camadas de manta. Assim foram fabricados corpos de prova que tinham a massa correspondente a duas camadas de manta e corpos de provas que tinham massa correspondente a três camadas de manta. Na Figura 3 são apresentadas as fibras particuladas utilizadas na fabricação dos corpos de prova. 


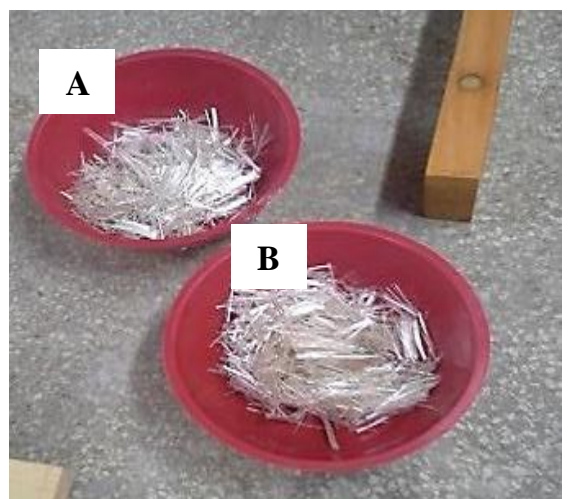

Figura 3. Fibras particuladas após o corte: $\mathbf{A}$ - massa correspondente a duas camadas de reforço; $\mathbf{B}$ - massa correspondente a três camadas de reforço.

Novamente foi colocada inicialmente uma camada de matriz. Em seguida as fibras particuladas foram dispostas de forma aleatória e com uma distribuição mais homogênea possível em cada camada. A matriz foi adicionada conforme novas camadas de reforço eram postas, até completar o molde.

Seguindo o mesmo procedimento, após 12 horas de cura os corpos de prova foram desmoldados, lixados, pesados e medidos.

\subsection{Avaliação da Massa Específica}

As dimensões foram obtidas utilizando um paquímetro, tendo um valor médio a partir de medidas em três pontos distintos. A partir dos dados obtidos de massa e dimensões dos corpos de prova confeccionados, primeiramente foram calculadas as massas específicas da forma convencional utilizando a equação 1 .

em que:

$$
\rho_{c}=\frac{M_{c}}{V_{c}}
$$

$\rho_{c}-$ massa específica do compósito

$M_{c}-$ massa do compósito

$V_{c}$ - volume do compósito

Com o objetivo de comparar os dados de massa específica calculados de forma convencional, foram realizadas análises das massas específicas em um picnômetro a gás (hélio), modelo Accupyc da Micromeritics, como apresentado na Figura 4. O método de picnometria a hélio consiste na determinação do volume de uma massa conhecida de amostra através da variação de pressão do gás em um volume calibrado. Para a realização dessas análises, amostras de aproximadamente $10 \mathrm{~mm}$ de comprimento foram retiradas dos corpos de prova. 


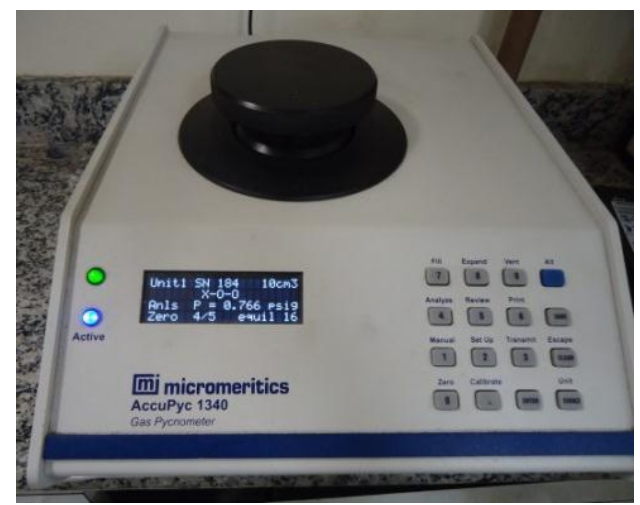

Figura 4. Picnômetro de gás hélio utilizado para determinação da massa específica

\subsection{Ensaio de tração direta}

Foram realizados ensaios de tração simples para a obtenção da tensão máxima de tração no material e o módulo de elasticidade longitudinal. Os ensaios de tração direta foram feitos em uma prensa universal de ensaios (Shimadzu Autograph ${ }^{\circledR}$ AG-X 100kN) com velocidade de 2,0 $\mathrm{mm} / \mathrm{min}$. Os ensaios foram realizados de acordo com as normas ISO 527-1(1993) [4] e ISO 5274 (1997) [3]. Na Figura 5 é apresentada a prensa utilizada nos ensaios de tração direta.

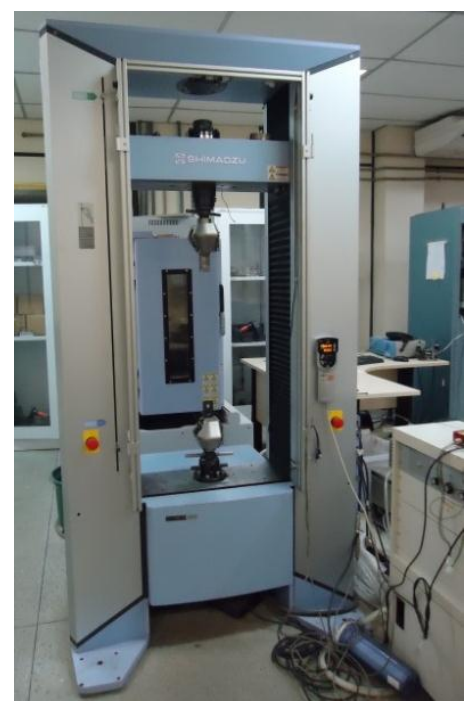

Figura 5. Prensa Shimadzu Autograph® AG-X $100 \mathrm{kN}$

A tensão de tração foi calculada a partir da carga de tração obtida pela célula de carga acoplada na Prensa Shimadzu.

A deformação foi calculada através da equação 2:

$$
\varepsilon=\frac{\Delta L}{L}
$$

em que:

$\Delta \mathrm{L}=$ alongamento medido através do LVDT's;

$\mathrm{L}=50 \mathrm{~mm}$, base de medida dos LVDT's (Linear Variable Differential Transformer) utilizados nos ensaios. 
O módulo de elasticidade longitudinal foi calculado a partir de dois valores de deformações $\varepsilon 1$ e $\varepsilon 2$ de acordo com a norma ISO 527-1(1993) [3]. O valor do modulo de elasticidade longitudinal foi obtido pela equação 3 .

$$
E=\frac{\sigma_{2}-\sigma_{1}}{\varepsilon_{2}-\varepsilon_{1}}
$$

em que:

$\sigma_{1}=$ tensão axial correspondente à deformação $\varepsilon_{1}=0,0005$;

$\sigma_{2}=$ tensão axial correspondente à deformação $\varepsilon_{2}=0,0025$;

A montagem dos corpos de prova na máquina de ensaio pode ser vista na Figura 6.

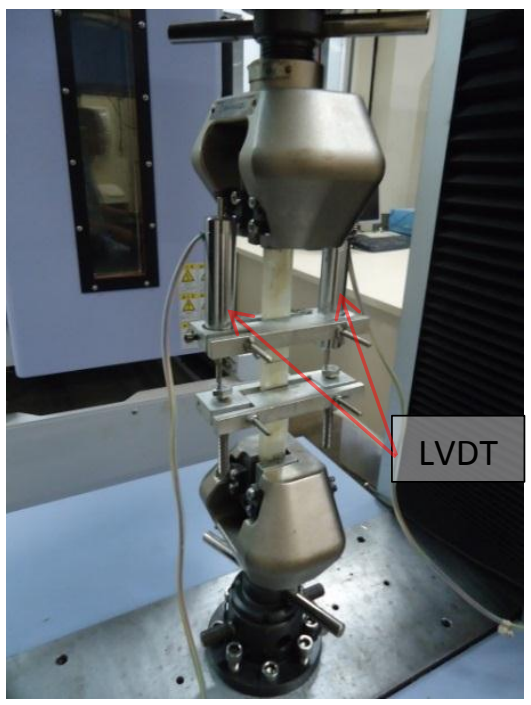

Figura 6. Montagem dos corpos de prova na máquina de ensaios.

\section{Apresentação e Discussão dos Resultados}

\subsection{Nomenclatura}

Na Tabela 1 é apresentada a nomenclatura para identificação dos ensaios realizados

Tabela 1. Nomenclatura dos ensaios realizados.

\begin{tabular}{|l|l|}
\hline Nomenclatura & Descrição \\
\hline CPR & \begin{tabular}{l} 
Corpo de prova de referência. \\
\hline CPM_02 \\
corpo de prova reforçado \\
com duas camadas de manta \\
de fibra de vidro.
\end{tabular} \\
\hline CPP_02 & $\begin{array}{l}\text { Corpo de prova reforçado } \\
\text { com três camadas de manta } \\
\text { de fibra de vidro. }\end{array}$ \\
\hline CPP_03 & $\begin{array}{l}\text { Corpo de prova reforçado } \\
\text { com fibras de vidro } \\
\text { particuladas com massa } \\
\text { equivalente a duas camadas } \\
\text { de manta. }\end{array}$ \\
$\begin{array}{l}\text { Corpo de prova reforçado } \\
\text { com fibras de vidro } \\
\text { particuladas com massa } \\
\text { equivalente a três camadas de } \\
\text { manta. }\end{array}$ \\
\hline
\end{tabular}




\subsection{Massa Específica do Compósito $\left(\rho_{c}\right)$}

Na Tabela 2 são apresentadas as médias das massas específicas dos compósitos calculados pela formula 2.1 e pelo picnômetro a gás. Foi possível observar uma diferença significativa entre os valores calculados e os obtidos pelo picnômetro a gás, chegando a uma variação de até $19.5 \%$. A diferença pode ser explicada pela imprecisão do procedimento manual, como por exemplo, os desvios e erros durante as medições dos corpos de prova. Na Figura 7 são apresentados os resultados das massas específicas, obtidas pelo picnômetro a gás. No gráfico estão apresentadas as médias e os desvios padrão das amostras realizadas.

Tabela 2. Massa Específica obtida pelo picnômetro a gás.

\begin{tabular}{|c|c|c|c|}
\hline CP & $\begin{array}{c}\boldsymbol{\rho} \mathbf{c}_{\text {cal. }} \\
\left(\mathbf{g} / \mathbf{c m}^{\mathbf{3}}\right)\end{array}$ & $\begin{array}{c}\boldsymbol{\rho} \mathbf{c}_{\text {pic. }} \\
\left(\mathbf{g} / \mathbf{c m}^{\mathbf{3}}\right)\end{array}$ & $\begin{array}{c}\boldsymbol{\rho} \boldsymbol{c}_{\text {pic }} \\
\boldsymbol{\rho} \boldsymbol{c}_{\text {cal }}\end{array}$ \\
\hline CPR & - & 1,1815 & - \\
\hline CPM_02 & 1.1269 & 1,2262 & 1.09 \\
\hline CPM_03 & 1.0838 & 1,2424 & 1.15 \\
\hline CPP_02 & 1.0304 & 1,2315 & 1.19 \\
\hline CPP_03 & 1.0746 & 1,2458 & 1.16 \\
\hline
\end{tabular}

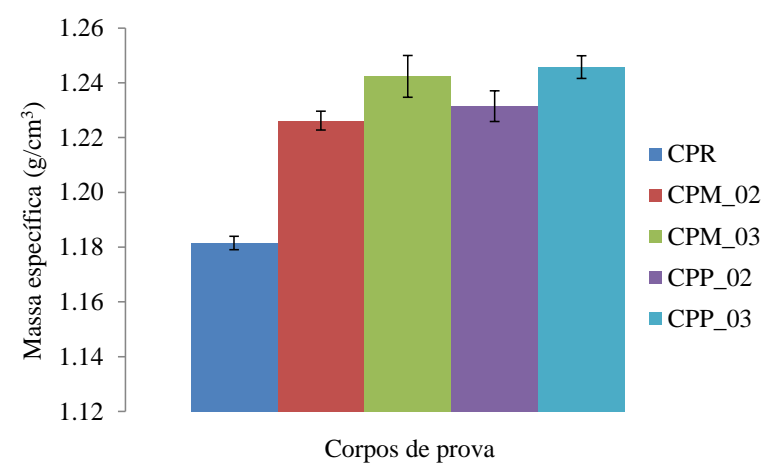

Figura 7. Resultados de massa específica dos compósitos.

Notou-se uma tendência de acréscimo da massa específica, com a adição do reforço fibroso. O compósito CPM_02 obteve uma massa específica de 3.8\% maior que o compósito de referência. Já o compósito COM_03 apresentou um aumento de sua massa específica em 5.2\%. Em relação aos compósitos produzidos com fibras particuladas, observou-se que as massas específicas se equipararam com as massas especificas dos compósitos reforçados com mantas, como esperado.

\subsection{Resistência à tração direta}

As curvas de tensão versus deformação dos compósitos fabricados são apresentadas na Figura 8. Vale salientar que as curvas apresentadas representam os valores médios obtidos nos ensaios experimentais. 


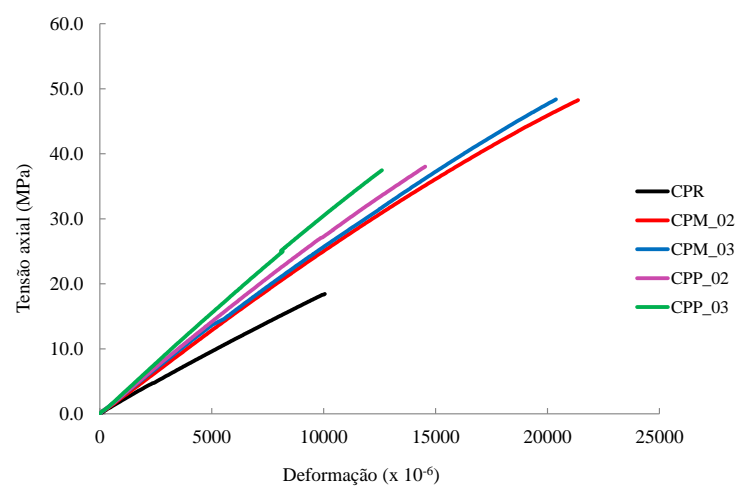

Figura 8. Curvas tensão versus deformação dos compósitos ensaiados.

Todos os compósitos apresentaram um comportamento praticamente linear. O reforço proporcionou um aumento de resistência à tração. Notou-se também que não houve um aumento significativo quanto ao número de camadas de manta de fibra e esse comportamento se repetiu para os compósitos com fibras particuladas.

Na Tabela 3 são apresentados os resultados de resistência à tração dos compósitos. São mostrados os valores médios e os coeficientes de variação obtidos.

Tabela 3. Resultados de resistência à tração (media e (coeficiente de variação (\%))).

\begin{tabular}{|c|c|}
\hline Compósito & Resistência à Tração $(\mathrm{MPa})$ \\
\hline CPR & $18.48(1.46)$ \\
\hline CPM_02 & $48.24(0.25)$ \\
\hline CPM_03 & $48.35(4.70)$ \\
\hline CPP_02 & $38.03(6.48)$ \\
\hline CPP_03 & $37.47(0.1)$ \\
\hline
\end{tabular}

O reforço fibroso aumentou de forma significativa a resistência à tração dos compósitos. Comparando os valores resistência a tração do compósito de referência (somente matriz) com os produzidos com mantas de fibras de vidro pode-se observar que este aumento foi de $161 \%$. Já em relação aos compósitos com fibras particuladas o amento foi de $105 \%$.

Em relação aos compósitos fibrosos, o uso de mantas de fibras de possibilitou um aumento na resistência à tração de aproximadamente $27 \%$.

Os valores obtidos (média e coeficiente de variação) de módulo de elasticidade dos compósitos são listados na Tabela 4.

Tabela 4. Resultados de módulo de elasticidade (media e (coeficiente de variação (\%))).

\begin{tabular}{|c|c|}
\hline Compósito & Módulo de Elasticidade (GPa) \\
\hline CPR & $1.92(1.94)$ \\
\hline CPM_02 & $2.58(0.16)$ \\
\hline CPM_03 & $2.77(4.80)$ \\
\hline CPP_02 & $2.92(11.19)$ \\
\hline CPP_03 & $3.17(3.28)$ \\
\hline
\end{tabular}


Diferentemente da resistência à tração, o módulo de elasticidade teve um ligeiro aumento com a variação da quantidade de camadas de fibras. Neste caso, os compósitos produzidos com fibras particuladas obtiveram maiores módulos de elasticidade.

Comparando os resultados do módulo de elasticidade do compósito de referência com o produzido com manta de fibra de vidro, notou-se um aumento de aproximadamente $44 \%$ (considerando o CPM_03). Já para o caso dos compósitos de fibras particuladas o aumento foi de 65\% (considerando CPP_03).

Os módulos de elasticidade dos compósitos particulados aumentaram cerca $14 \% \mathrm{em}$ relação aos compósitos produzidos com mantas de fibras de vidro.

São mostrados, nas Figuras 9 e 10, corpos de prova (referência e com reforço fibroso) após terem sido ensaiados à tração direta.

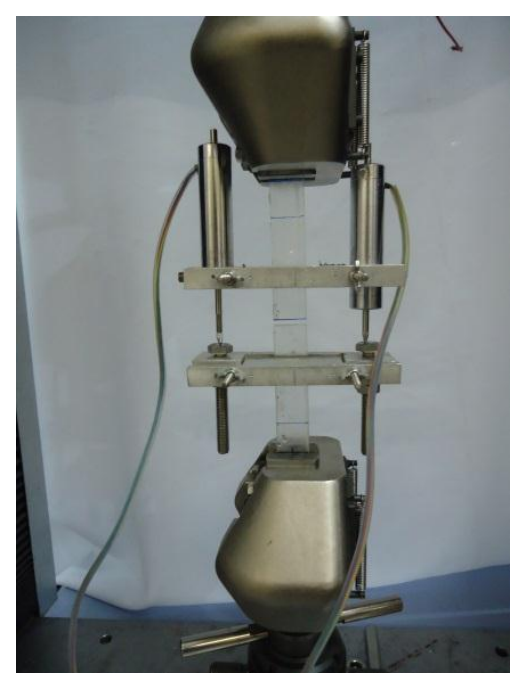

Figura 9. Ruptura Matriz

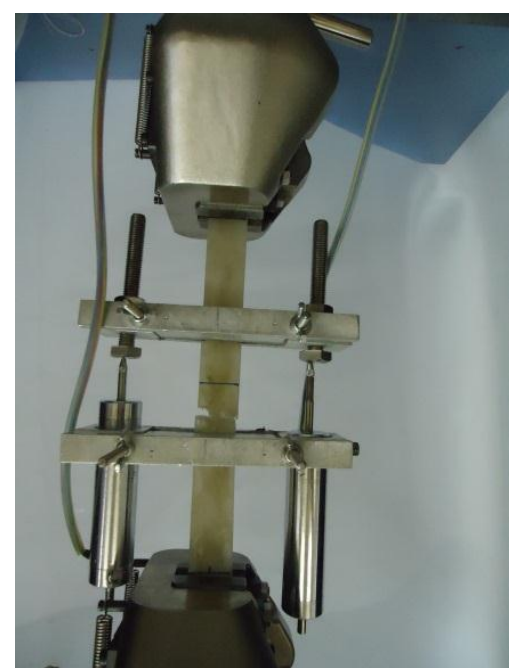

Figura 10. Ruptura de compósito reforçado com fibras 


\section{Conclusão}

Os resultados apresentados neste trabalho atingiram a proposta inicial de realizar um estudo experimental para enriquecer o conhecimento a cerca dos materiais compósitos.

Os resultados obtidos mostraram a eficiência de um reforço fibroso, aumentando significantemente a resistência à tração e o módulo de elasticidade.

Também foi possível observar que os corpos de prova reforçados com manta apresentaram melhores resultados de resistência à tração, em razão da melhor distribuição de suas camadas no corpo de prova e uniformidade das fibras em cada camada. Os reforçados com fibras particuladas, inevitavelmente, possuíam aglomerações de fibras, deslocamento das fibras para camadas inferiores, vazios devido à inserção de ar durante o processo de moldagem.

No processo experimental desenvolvido, o número de camadas de reforços com fibras não alterou a resistência à tração dos compósitos, mas, houve alteração em relação ao módulo de elasticidade.

\section{Agradecimentos}

Os autores agradecem ao Laboratório de Estruturas e Materiais Prof. Lobo Carneiro da COPPE/UFRJ pela disponibilização dos equipamentos para a execução do programa experimental.

\section{Referências Bibliográficas}

NIU, Michael Chun-yung. Composite airframe structures: Pratical Design Information And Data. 1. ed. Hong Kong: Conmilit Press Ltd., 1992.

BANK, L. C. Composites for Construction: Structural Design with FRP Materials. New Jersey, John Wiley \& Sons, 2006.

ISO 527-4. "Determination of tensile properties of plastics Part 4: Test conditions for isotropic and orthotropic fibre-reinforced plastic composites". International Standard, 1997.

ISO 527-1. "Plastics - Determination of tensile properties -Part I: General principles". International Standard, 1993.

ISO 13003. "Fibre-reinforced plastics - Determination of fatigue properties under cyclic loading conditions". International Standard, 2003. 